\title{
Postoperative Chylothorax: Is Octreotide A Valid Therapy?
}

\author{
Owais TA*, Back D, Girdauskas E and Kuntze T
}

Department of cardiac surgery, Zentralklinik Badberka, Thüringen, Germany

*Corresponding author: Owais TA, Zentralklinik Badberka, 99437, Badberka, Germany, Tel: +49-036458181155, 01622397476, E-mail: tamerowaiss1976@yahoo.com

Citation: Owais TA, Back D, Girdauskas E, Kuntze T (2015) Postoperative Chylothorax: Is Octreotide A Valid Therapy? J Case Rep Stud 3(3): 307. doi: 10.15744/2348-9820.3.307

Received Date: February 18, 2015 Accepted Date: June 10, 2015 Published Date: June 12, 2015

\begin{abstract}
Chylothorax is an extremely rare and serious complication of cardiac surgery, with a poor outcome unless treated at the right time and in the right way. We report a case of a 76-year-old female who developed chylothorax after coronary artery bypass grafting. The chylothorax was successfully treated in 7 days by octreotide administration and medium-chain triglycerides enriched diet. Octreotide, a long-acting somatostatin analogue, is an effective, valid and safe drug for the treatment of postoperative chylothorax and may highly reduce the need for reoperation.
\end{abstract}

Keywords: Anesthesia; Mediastinum; Pleura; Coronary disease

\section{Introduction}

In September 2013, a 76-year-old female was presented to our outpatient clinic with dyspnea and rapid progressive loss of weight (5 kilograms since discharge from the hospital). Fifteen days earlier, she was operated upon for CABG (coronary artery bypass grafting) for triple-vessel disease. The operation was done via median sternotomy on heart-lung machine where left internal mammary artery was anastomosed to the left anterior descending artery and saphenous venous grafts to the obtuse marginal artery and the posterior descending artery as well. The operative and postoperative course was eventless and smooth. On physical examination, diminished air entry on the left hemithorax was concluded and obvious signs of fatigue and debilitation were noticed such as dry shrinked skin, muscle wasting and loss of weight. Chest radiography revealed a left-sided pleural effusion and mediastinal shift through the contralateral side (Figure 1). A pleural intercostal tube was inserted in the fifth left intercostal space in the midaxillary line immediately on admission, and 2,100 $\mathrm{mL}$ of chylous fluid was drained by thoracentesis. Chemical laboratory analysis of the pleural fluid revealed a total cholesterol level of $23 \mathrm{mg} / \mathrm{dL}$; triglyceride $237 \mathrm{mg} / \mathrm{dL}$; glucose $79 \mathrm{mg} / \mathrm{dL}$; protein $35 \mathrm{mg} / \mathrm{dL}$, without bacterial growth. On the basis of these findings, a diagnosis of chylothorax was made.

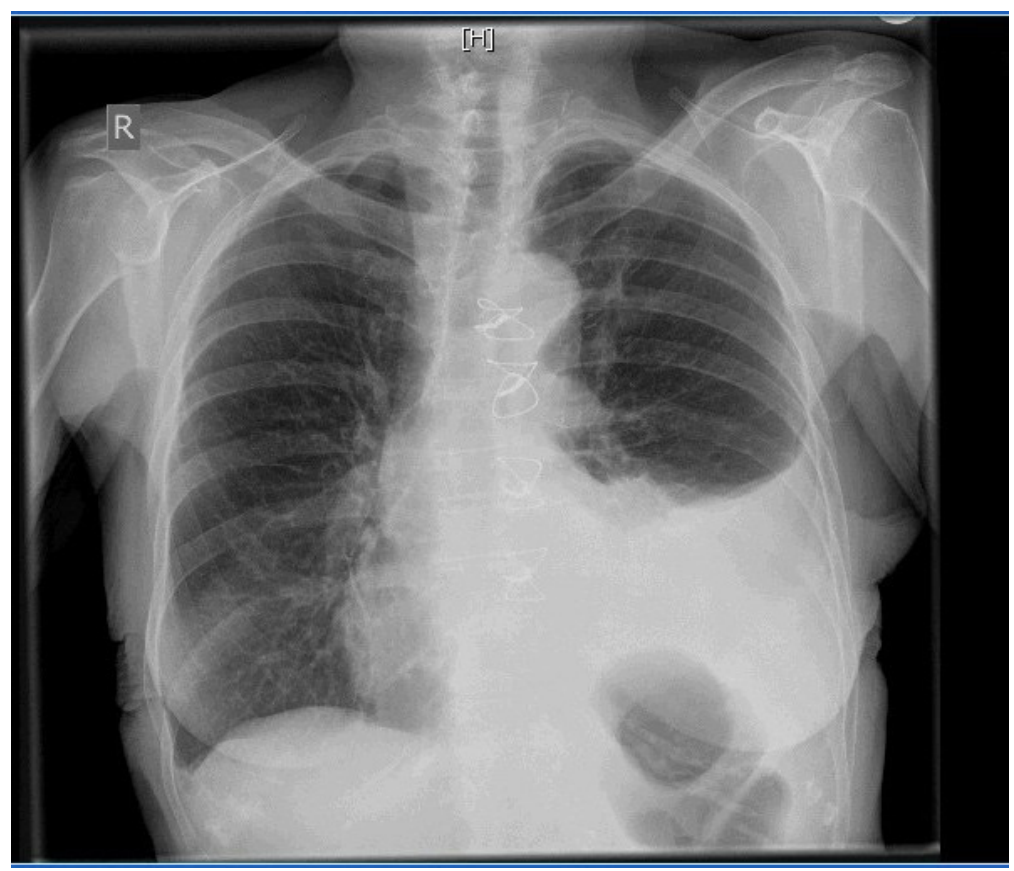

Figure 1: Xray on admission 
On the same day of admission and intercostal tube insertion, an oral diet that contained medium-chain triglycerides (MCT) was begun and parentral nuitrition substitution as well. Because of continuous, extensive drainage for 48 hours, the decision was made to administer subcutaneous octreotide $100 \mu \mathrm{g}$ every 8 hours. The chylous fluid drainage, which reached $850 \mathrm{~mL} /$ day on the day before octreotide therapy, decreased to $300 \mathrm{~mL} /$ day on the 2 nd day after the initiation of octreotide therapy. After 3 days of therapy, octreotide was stopped because of the good satisfactory response concerning drainage, X-ray findings (Figure 2) and gradual increase of weight in favour of the dietary protocol (4 kilograms in 6 days), and we decided to remove the intercostal tube on the 7th day-after pleural drainage had become serous and had decreased to less than $50 \mathrm{~mL} /$ day. The patient was discharged on the 7 th day of treatment, with satisfactory X-ray. With instructions to continue the diet that contained MCT. After 1 months of followup, the patient was on a normal oral dietary protocol, and the chest X-ray was free from residual effusion. At her second month of follow-up, the patient was fine, regained 6 kilogram weight, ambulant and without clinical or radiological signs of recurrence of chylothorax.

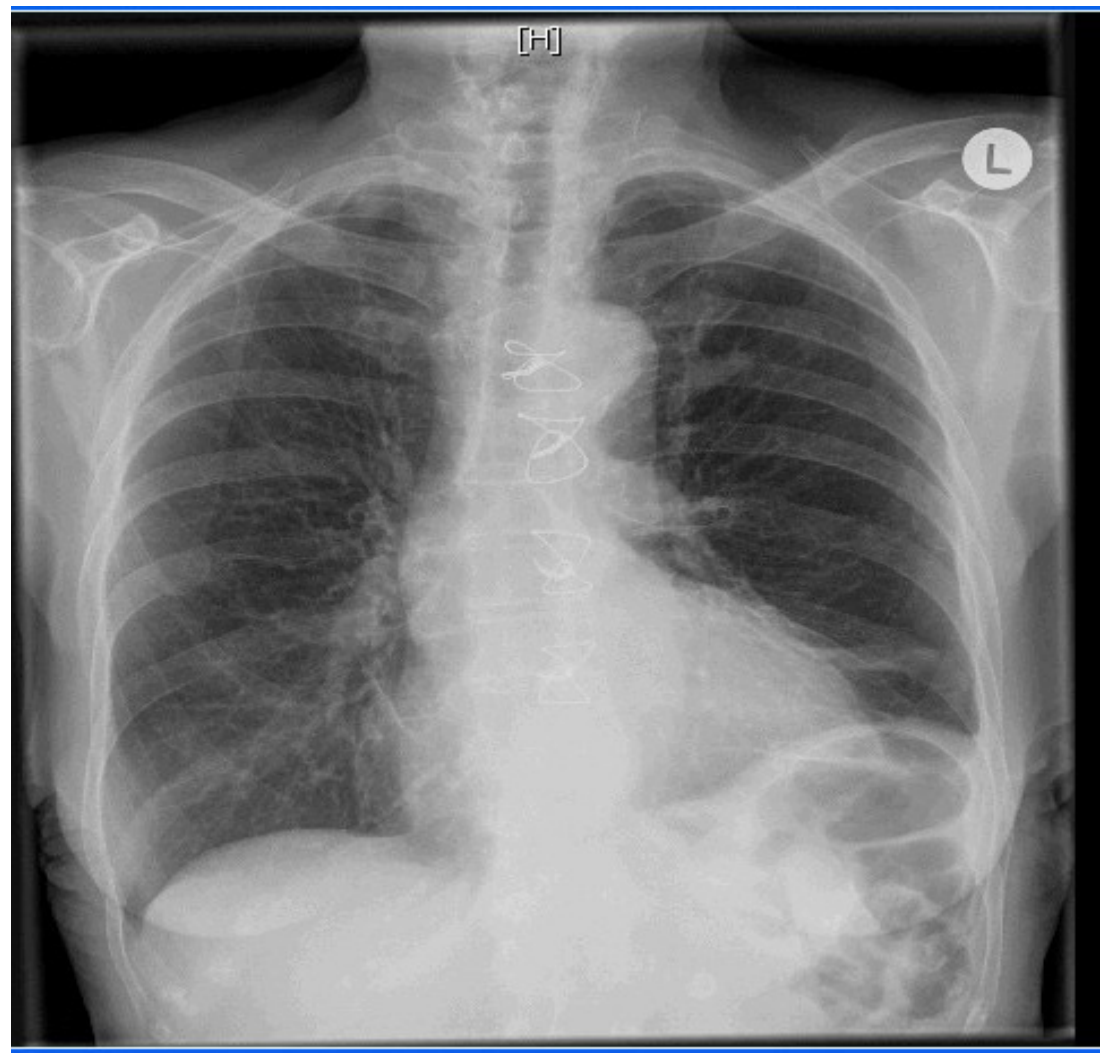

Figure 2: Xray after 3 days of management with sandostatin

\section{Discussion}

Postoperative chylothorax is a major and rare life-threatening postoperative complication, with an occurrence incidence of $0.2 \%$ to $1.4 \%$ in cardiothoracic operations [1]. Injury of the the thoracic duct or the adjacent small lymphatic channels is commonly met with esophagectomy, mediastinal exenteration, or pediatric cardiac operation. Post CABG (coronary artery bypass grafting) chylothorax is usually related as a consequence of internal mammary artery harvesting in adult patients. Precisely in our department, postoperative chylothorax has been observed in 1 of 830 patients who underwent CABG. Routine treatment of chylothorax entails the insertion of an intercostal pleural tube, total parenteral nutrition, and duct ligation via a transthoracic surgical approach. Minimally invasive techniques such as video-assisted thoracoscopic surgery (VATS), introduced in the 1990s, have replaced conventional surgical approaches and have been used successfully in clipping of the thoracic duct and in the application of fibrin glue and thus excluding the major expected complications of open surgical procedures.

Other theraperutic models as transthoracic and transabdominal duct ligation, percutaneous thoracic duct embolization and pleuroperitoneal shunt are usually indicated in cases of progressive chylous drainage (of 1 to 2 weeks' duration, with output greater than $1,000 \mathrm{~mL} /$ day) [2]. The use of thoracic duct embolization has been very encouraging due being less invasive, good results and reduction in the need for repearted surgeries [3]. Surgical outcomes are not always satisfactory and lead to a relatively high morbidity and mortality rates. Because chylothorax leads to critical losses of fluid, lymphocytes, proteins, coagulation factors, and antibodies, and because patients who develop chylothorax generally are in poor physical condition, noninvasive, conservative treatment may be valuable in this population. Cerfolio and colleagues state an incidence of reoperation in 34 of 47 patients diagnosed with chylothorax [1]. In 23 of the 34 (68\%), injury to the thoracic duct was discovered at reoperation. Complications occurred in 18 of the 34 , and 3 of those required a 2 nd reoperation. 
Few agents other than octreotide have been used for conservative treatment of postsurgical lymphatic leak [4].OK-432 (Su-strain treated Streptococcus pyogenes) causes chemical pleurodesis and have been used in only in few patients with success [4]. Intravenous etilefrine hydrochloride decreases chyle production by a sympathomimetic effect on smooth muscle fibers of the lymphatic vessels. It has been used in 10 patients and was successful in 8 of them [5].The other 2 patients required surgical intervention, after inadequate decreases in chyle drainage. Intravenous etilefrine hydrochloride was stopped in 1 patient due to interactions with other sympathomimetic drugs [5].

Octreotide is a long-acting somatostatin analogue. It acts directly on vascular somatostatin receptors and reduces lymph fluid excretion. Moreover, by increasing splanchnic arteriolar resistance and decreasing gastrointestinal blood flow, octreotide indirectly reduces lymphatic flow. Some other theories are: octreotide blocks pancreatic and biliary secretion by inhibiting serotonin and other gastrointestinal peptides [5].

Recently, many investigators reported successful treatment of chylothorax post pediatric cardiac operations with somatostatin and its analogue [6,7]. There are only a few reports of its use in adults.

We have used ocreotide in our adult chylothorax patients with the satisfactory good results, reaching a remakable decrease in chyle drainage within 3 days of the onset of therapy. To the best of our knowledge, no failure or recurrence in the treatment of chylothorax with octreotide therapy has been reported in the literature. However Potential side effects of octreotide therapy are fluid retention, hyponatremia, hypokalemia, stomachache, headache, nausea, vomiting, tympanitis and epistaxis. we faced no side effects in our patient. We did avoid Surgical intervention. We insisted on using a middle chain triglyceride diet, because it is transported directly into the portal system, bypassing the lymphatic system, thus reducing lymphatic flow through the thoracic duct. Ocreotride administration may be used as the initial conservative therapy for chylothorax. A surgical approach has been suggested in adults after a traumatic event if the daily loss of chyle over a 5-day period exceeds 1,000 mL/day [1]. Thoracoscopic surgery may be the 1st choice; in the event of failure, open surgery may be performed in the same session.

In conclusion, octreotide is a valid, effective, noninvasive therapeutic modality in postoperative chylothorax. It should be respected as a therapeutic modality for treatment of chylothorax post cardiac surgery. Because it may reduce the need for surgery, further studies with increased numbers of patients are recommended to evaluate these results.

\section{References}

1. Cerfolio RJ, Allen MS, Deschamps C, Trastek VF, Pairolero PC (1996) Postoperative chylothorax. J Thorac Cardiovasc Surg 112: 1361-6.

2. Valentine VG, Raffin TA (1992) The management of chylothorax. Chest 102: 586-91.

3. Marthaller (2015) Therapeutic options in treatment of postoperative Chylothorax. Ann J Surg 209: 235-9.

4. Nakano A, Kato M, Watanabe T, Kawai N, Ota H, et al. (1994) OK-432 chemical pleurodesis for the treatment of persistent chylothorax. Hepatogastroenterology 41: 568-70.

5. Guillem P, Papachristos I, Peillon C, Triboulet JP (2004) Etilefrine use in the management of post-operative chyle leaks in thoracic surgery. Int Cardiovasc Thorac Surg 3: 156-60.

6. Hamdan MA, Gaeta ML (2004) Octreotide and low-fat breast milk in postoperative chylothorax. Ann Thorac Surg 77: 2215-7.

7. Bryant (2014) Recent techniques in management of chylothorax. Ann Thoracic surgery 98: 232-5.

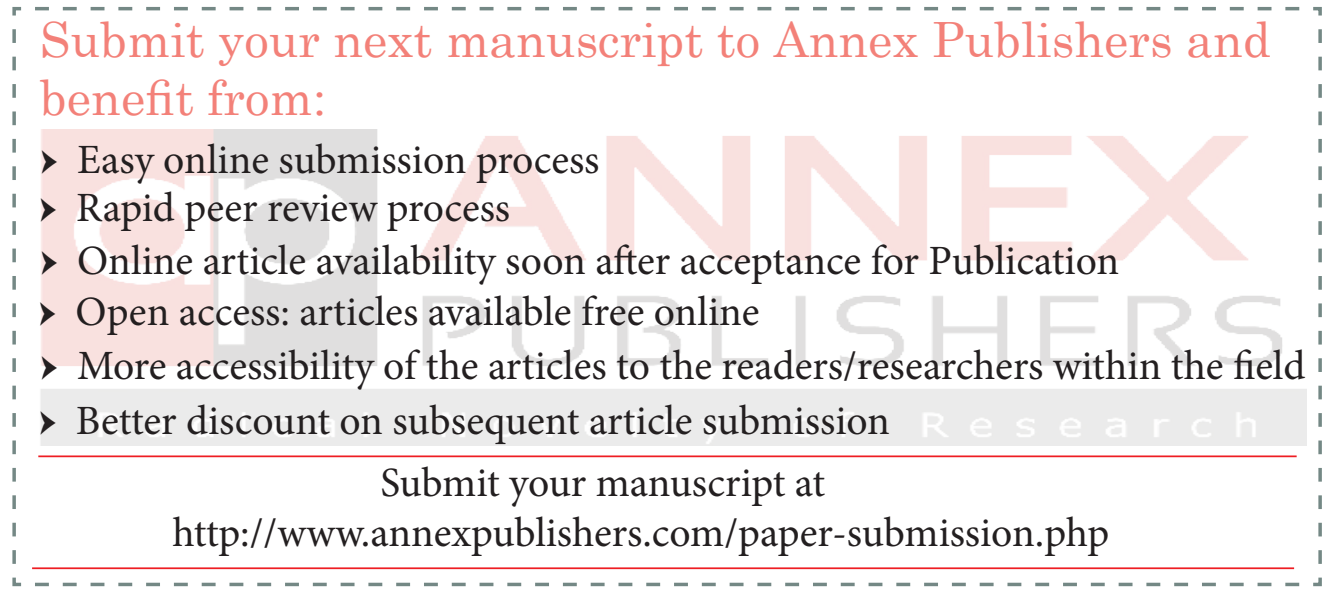

